\title{
Variabilité Climatique Et Changements Dans L'environnement À Korhogo En Côte D'ivoire : Mythes Ou Réalité ?
}

\author{
Boko-Koiadia Adjoua N. Nadège \\ Doctorante à l’Institut d'Ethnosociologie (IES) à l’Université Félix \\ Houphouët-Boigny de Cocody-Abidjan, Côte d'Ivoire \\ Centre Suisse de Recherches Scientifiques (CSRS) en Côte d'Ivoire \\ Guéladio Cissé \\ Professeur à l'Université de Bâle, \\ Institut tropical et de santé publique suisse (Swiss TPH)

\section{Brama Koné} \\ Enseignant chercheur à l’Université Péléforo Gon Coulibaly de Korhogo, \\ Côte d'Ivoire \\ Centre Suisse de Recherches Scientifiques en Côte d'Ivoire

\section{Dedy Séri} \\ Professeur, Université Félix Houphouët Boigny de Cocody-Abidjan, \\ Côte d'Ivoire
}

\begin{abstract}
Climate change which is considered as a threat to biodiversity, causes paradoxical situations everywhere it manifests itself. These include, among others, drought and desertification; floods and storms; sea level rise, reduction of the vegetation cover; water supply sources and, animal and plant species disappearance. In Korhogo, in the North of Côte d'Ivoire, the climate variability and environmental change phenomena are perceived and described by the population. A qualitative approach with historical and comparative analysis tools are used in this study to focus on the perceptions of the populations through indicators of both climate variability and environmental changes. It also analyses the major challenges the population will face, which are mainly related to alteration in farming practices and seasons, loss of production, and extension of the lean period, the risk of food insecurity and conflicts related to the exploitation and the distribution of water resources; and mostly, to the lack of information- educationcommunication on the phenomenon. On the whole, although the study brings out an awareness about climate variability and environmental changes, it
\end{abstract}


especially show the need for emergent multidimensional actions for effective response strategies to the changes observed in the area

Keywords: Climate variability, characterization, perception, Côte d’Ivoire, West Africa

\section{Résumé}

Considéré comme une menace de la diversité biologique, le changement climatique provoque des situations paradoxales partout où il se manifeste. Ce sont entre autres: la sécheresse et la désertification; les inondations et les tempêtes; l'élévation des niveaux des mers; la réduction du couvert végétal; la disparition des sources d'approvisionnement en eau, la disparition d'espèces animales et végétales. A Korhogo, au nord de la Côte d'Ivoire, le phénomène de la variabilité climatique et des changements environnementaux sont perçus et décrits par la population. Par une approche qualitative avec les outils d'analyse historique et comparative, l'étude met en exergue les perceptions des populations à travers les indicateurs aussi bien de la variabilité climatique que des changements dans l'environnement. Elle analyse également les défis majeurs auxquels la population doit faire face et qui sont liés essentiellement au bouleversement des pratiques agricoles et des cycles culturaux, à la baisse de la production et de l'allongement de la période de soudure, au risque d'insécurité alimentaire et de conflits liés à l'utilisation et au partage des ressources en eau. Mais surtout, au déficit d'information-éducation-communication sur le phénomène. En définitive, même si l'étude révèle une prise de conscience de la variabilité climatique et des changements dans l'environnement, elle montre surtout l'urgence qu'il y a à mener des actions multidimensionnelles pour d'efficaces stratégies d'adaptation aux changements perçus dans la zone.

Mots-clés: Variabilité climatique, caractérisation, perception, Côte d’Ivoire, Afrique de l'Ouest

\section{Introduction}

Depuis la fin des années 1960, la Côte d’Ivoire, comme l'ensemble des pays de l'Afrique de l'Ouest et Centrale, connaît une aggravation de la variabilité climatique qui se manifeste, en particulier, par une modification du régime des précipitations et par une diminution des hauteurs annuelles (Brou et al., 2005). En Côte d'Ivoire, une régression statistique concernant la pluviométrie a relevé que les précipitations annuelles auraient diminué en moyenne de $0.5 \%$ par an entre 1965 et 1980; une diminution qui se serait élevée à 4,6\% dans les années 1980 (Ministère de l'environnement et de la forêt, 1999) 
Pis, le pays est confronté à des problèmes environnementaux d'origines diverses telles que la disparition du couvert forestier, la disparition de la faune, l'appauvrissement des sols, la pollution et eutrophisation des eaux, la pollution de l'air, la dégradation du lieu urbain, la persistance des maladies environnementales (Le diagnostic du Livre Blanc de l'Environnement de Côte d'Ivoire, 1995).

En effet, la forêt ivoirienne qui couvrait 16 millions d'hectares en 1900, 12 millions d'hectares en 1956 et 9 millions d'hectares en 1965, ne représentait plus que 2,5 millions d'hectares en 1991, soit un taux de déboisement de l'ordre de 250000 d'hectares par an sur la période de 19651991 (MECU, 1991). Elle connaît un des taux les plus élevés de déforestation au monde; soit une perte de plus de $67 \%$ du couvert forestier initial depuis 1960 (Koné, 2011).

Selon N'Guessan et al. (2006), pendant longtemps, plusieurs auteurs dont Lanly (1969), sodefor (1976), Arnaud et Sournia (1980), Bertrand (1983), Mecv (1991), MINEF (1998) et la FAO (2000), ont signalé les actions anthropiques de dégradation du couvert forestier que sont l'expansion de l'agriculture, l'agriculture sur brûlis, les feux de brousse, l'utilisation marginale d'intrants et l'utilisation des ligneux à des fins diverses (pharmacopée, alimentation, construction d'habitat traditionnel, artisanat).

Toutes ces actions anthropiques et l'absence de protection de la biodiversité ont contribué non seulement à dégrader les surfaces forestières, entraînant une diminution de la couverture ligneuse mais, à menacer dangereusement les équilibres du patrimoine faunique et floristique (Yao, 2005). Ce qui est certain, c'est que la déforestation brutale a conduit à une "savanisation" du pays renforcée avec l'harmattan, vent sec du Nord (Commission Européenne, 2006). Aujourd'hui, le tiers de la superficie de la Côte d'Ivoire (environ 100000 km2 sur 320000 km2) est occupé par la savane (N’Guessan et al., 2006).

Le Nord de la Côte d'Ivoire n'est pas épargné par cette transformation de la végétation naturelle et les problèmes environnementaux que cela provoque.

En effet, la zone de Korhogo a connu également une déforestation à grande échelle avec la construction de barrages visant à accroître le potentiel rizicole (SODERIZ), la création d'un complexe agro-industriel (sucre) à Ferkéssédougou (SODE-SUCRE), l'extension de la zone cotonnière et développement de la culture attelée (C.I.D.T., projet Banque Mondiale).

Ainsi, l'industrialisation de l'agriculture et l'avènement des cultures de rentes telles que le coton, l'anacarde, les vergers, etc. ont contribué à la déforestation de la zone. Sa végétation de type savanes arbustives et de forêt galeries, est durement marquée par cette déforestation. Aussi, dans la 
décennie 2000- 2010, la zone de Korhogo a-t-elle connu des évènements climatiques extrêmes qui sont: une sécheresse en 2004-2005 avec l'assèchement des points d'eau (barrage, rivières, puits, etc.) et une inondation en 2006-2007 avec beaucoup de destructions et des dommages (pertes en vies humaines et des blessés physiques; destruction de ponts, de nombreuses habitations et des cultures maraîchères faites dans le lit du barrage, etc.)

Le présent papier vise la mise en évidence de la perception de la variabilité climatique et des changements dans l'environnement par les populations de Korhogo. Il s'agit de décrire les indicateurs de changement tant climatiques qu'environnementaux dans un premier temps et ensuite, identifier les défis face à ce phénomène. Enfin, proposer des solutions face à ces observations.

\section{Méthodologie}

\section{Zone de l'étude}

L'étude a été effectuée à Korhogo, ville située au Nord de la Côte d'Ivoire qui compte environ 212.546 habitants. Elle fait partie de la région des savanes et est frontière au Mali et au Burkina Faso. Cette ville est à 600 km d' Abidjan, capitale économique de la Côte d'Ivoire. Sa végétation est de type savanes herbeuses, arbustives ou arborées, de forêt galeries et de forêts claires. La population de Korhogo, constituée principalement de sénoufos et de malinkés (Dioula), est à majorité animiste et vit essentiellement de l'agriculture. Le département de Korhogo est situé dans la zone de climat soudanais et il est marqué par une alternance de deux (2) saisons:

- La saison sèche, très marquée par l'harmattan entre décembre et janvier et des pointes de chaleur en mars et avril, s'étend de novembre à avril;

- La saison des pluies s'étend de mai à octobre avec des pluviométries maximales en juillet et août. Les températures moyennes varient entre $24^{\circ}$ et $33^{\circ} \mathrm{c}$. Les mois les plus chauds sont février, mars et avril avec $36^{\circ} \mathrm{c}$ et les mois les plus frais sont décembre et janvier avec $16^{\circ} \mathrm{c}$ avec une moyenne pluviométrique annuelle se situant entre $1100 \mathrm{~mm}$ et $1600 \mathrm{~mm}$. 


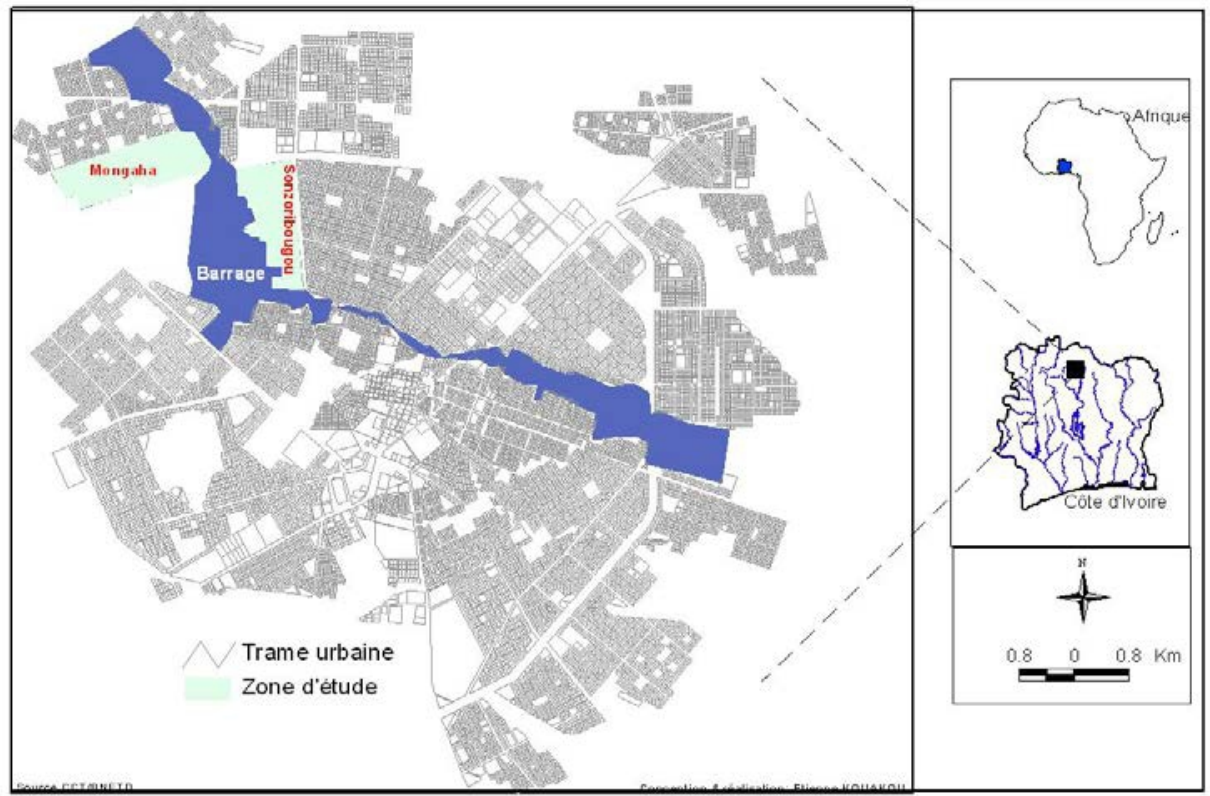

Figure 1 : Carte de la zone d'étude (Source : Boko, 2013)

\section{Collecte des données}

Une approche qualitative est adoptée dans le cadre de cette étude avec différents outils de collecte des données.

L'étude est basée sur une approche socio-anthropologique qui permet de capter la perception des populations concernant les variabilités du climat et les changements dans l'environnement. Les données de l'étude ont été collectées grâce à des entretiens semi-directifs individuels et de groupe (focus group), des histoires de vie, des interviews et des observations directes.

\section{Entretiens semi-directifs, focus group et histoires de vie}

Les entretiens semi-directifs individuels et de groupe (focus group), les histoires de vie et interviews ont été réalisés entre Mai 2009 et Février 2012, en zone urbaine (Korhogo ville et quartiers autour du barrage) et périurbaine (quatre villages à proximité de Korhogo). Le choix des quartiers (Mongaha, Haoussabougou et Sonzoribougou) se justifie non seulement par le fait que ces habitants mènent des activités autour du barrage mais, ceux-ci sont les premières victimes des événements climatiques extrêmes (inondations). En ce qui concerne les quatre autres villages (Gbondokaha, Longnokaha, Kounontonvogo et Natiokobadara), la pré-enquête a permis de noter qu'ils ont enregistré (les trois premiers villages) des pertes importantes pendant les grands moments de pluie et le quartrième village est habité en grande partie par des «Fodonons ou faiseurs de pluies». Ont été interrogé au 
cours de cette étude, des chefs traditionnels garants de l'histoire et de la tradition en pays Sénoufo, des acteurs de l'administration publique et privée locale et internationale, des personnes du troisième âge, des jeunes, des intellectuels et des non intellectuels, des femmes. Au total 44 entretiens individuels et 12 entretiens de groupe ont été effectués. Les guides d'entretiens ont été élaborés en fonction des acteurs et du type d’information recherchée.

En effet, les entretiens semi-directifs ont été fait pour identifier les indicateurs de la variabilité climatique et des changements dans l'environnement; ainsi que les conséquences vécues de ces changements. Des interviews pour avoir des données plus pointues sur le phénomène dans la zone, les actions d'aide entreprises par les structures privées et publiques et les stratégies d'adaptation conseillées par celles-ci pour permettre à la population de faire face au phénomène. Les récits de vie ont permis d'avoir des informations sur l'évolution du climat et de la végétation dans la zone d'étude mais aussi, des changements observés dans les différents domaines d’activités des acteurs au fil des années. Enfin, des focus group pour avoir une vue plus générale de la connaissance, des conséquences et des difficultés de la population face aux phénomènes de la variabilité climatique et des changements dans l'environnement.

Les guides tournaient autour des thèmes suivants: Informations générales sur l'environnement socio-culturel et politique du peuple Sénoufo, les indicateurs de changements au niveau du climat et de l'environnement, les effets des changements sur la population et leurs activités, les stratégies endogènes d'adaptation et les propositions de solutions pour faire face au phénomène.

\section{Observations directes et photographie}

Après ou au cours des entrevues, une observation directe des sites est organisée pour vérifier de visu les informations données. Cette observation directe s'est faite pendant la saison sèche et pendant la saison des pluies sur des sites sélectionnés. Elle a permis de vérifier et/ou confronter les informations collectées lors des entretiens sur les effets de la variabilité climatique sur l'environnement. Ces sites d'observation sont les sources d'eau (puits, rivières, barrages, ponts, marigots, bas-fonds, parcelles d’activités de maraîchage, etc.). L’outil principal de l'observation est la photographie.

\section{L’analyse des données}

Deux méthodes d’analyse ont été utilisées: la méthode historique et la méthode comparative.

- La théorie de la modernisation et celle de la dimension anthropique 
du réchauffement climatique stipulent que l’origine de la crise écologicoclimatique se trouve dans le mode de développement industriel et donc dans les actions antérieures de l'homme sur son environnement. Ainsi, l'explication des problèmes environnementaux et climatiques actuelle ne se trouve pas au présent mais, au passé. C’est pourquoi, la méthode historique est ici privilégiée pour comprendre et expliquer les causes actuelles des phénomènes de superposition climatiques et de changement dans l'environnement à Korhogo. Une analyse de l'évolution historique de la biodiversité et du climat dans la zone de Korhogo, ainsi que des activités principales dans la zone permet de comprendre et de situer les transformations subies.

- La méthode comparative

Cette méthode permet d'analyser le discours déjà comparatif des populations en rapport avec l'environnement, le climat et leurs différentes activités. Cet outil a permis de mettre en évidence les changements et bouleversements observés, mais surtout, de se rendre compte de la gravité et de l'urgence des conséquences des problèmes environnementaux et climatiques dans la zone de Korhogo.

\section{Résultats}

\section{Perceptions et observations des changements dans l'environnement à Korhogo \\ Bouleversements observés avant et après 1970}

A l'instar de l'Afrique Subsaharienne, qui a connu de réelles perturbations climatiques et hydrométriques au début des années 70, les années 80 ont particulièrement marqué les populations de Korhogo selon les données collectées sur les évènements climatiques extrêmes. En effet, aussi bien en entretien semi-directif, en histoire de vie comme en focus group, les bouleversements climatiques intervenus au cours de ces années ont été indiqués avec précision par les enquêtées. Aussi, l’analyse historique des données d'enquête indique -t'elle que la région de Korhogo a toujours eu deux grandes saisons pluviométriques dans l'année: la saison sèche et la saison des pluies d'une durée de six mois chacune comme le précise cette citation représentative de la perception générale des enquêtés. "Initialement, chez nous c'était six (06) mois de saison sèche et six (06) mois de saison pluvieuse » (Entretien individuel /chef du village de Kounontovogo, 06 mai 2010). Ce qui ne semble plus être le cas actuellement selon les constats et observations des populations. La totalité des enquêtés soutient que la zone connaît aujourd'hui des perturbations dans la durée et la période des saisons. Le Tableau1 indique ces changements. 
Tableau 1 : Caractérisation des périodes et durée des saisons avant

et après 1970

\begin{tabular}{|c|c|c|}
\hline & Avant les années 70 & Après les années 70 \\
\hline $\begin{array}{l}\text { Saisons } \\
\text { pluvieuses }\end{array}$ & 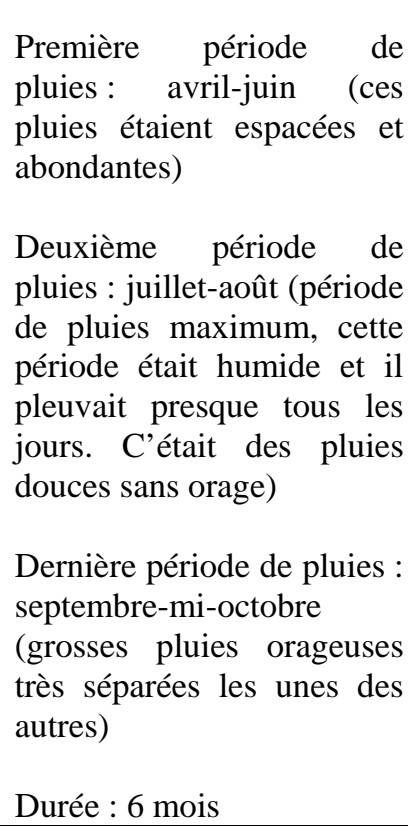 & $\begin{array}{l}\text { NB : Système de pluies très perturbé et } \\
\text { complexe car les périodes et la durée } \\
\text { changent en fonction des années. } \\
\text { Fin février- début mai : Premières pluies } \\
\text { marquant le début de la saison pluvieuse } \\
\text { juillet- août: Période de pluies douces et } \\
\text { fréquence comme par le passé } \\
\text { Septembre : Ce mois est de plus en plus } \\
\text { marqué par des pluies douces et non } \\
\text { orageuses. Il pleut maintenant plus, au cours } \\
\text { de ce mois qu'en août. } \\
\text { De fin septembre à octobre : Pluies orageuses } \\
\text { et espacées. } \\
\text { Durée : } 6 \text { mois, mais de façon discontinue et } \\
\text { incertaine. }\end{array}$ \\
\hline $\begin{array}{l}\text { Saisons } \\
\text { sèches }\end{array}$ & $\begin{array}{l}\text { De la mi-octobre au mois } \\
\text { de mars sans possibilité de } \\
\text { pluies } \\
\text { Durée : } 6 \text { mois }\end{array}$ & $\begin{array}{l}\text { De novembre à début février avec possibilité } \\
\text { de pluies isolées } \\
\text { mai- juin : Période de sécheresse se } \\
\text { prolongeant souvent jusqu'aux premières } \\
\text { semaines de juillet. } \\
\text { Durée : } 6 \text { mois et demi }\end{array}$ \\
\hline
\end{tabular}

Il ressort de ce tableau que les pluies ne sont plus régulières depuis les années 1980. Elles commencent timidement et de façon discontinue au cours des mois de février à mai en marquant des arrêts lors des mois de juinjuillet (juin et les premières semaines de juillet faisant partie de la saison sèche) pour prendre fin en octobre. Pour les enquêtés, il est désormais impossible de savoir exactement le nombre de mois de pluie. Pendant que la majorité des enquêtés parlent simplement de décalage des saisons, d'autres évoquent cinq, quatre, voire trois mois de pluies en moyenne par an maintenant. Des témoignages attestent des changements et bouleversements au niveau climatique: "Avant, les pluies commençaient pratiquement au mois d'avril, peut-être mars même, et les gens commençaient à faire les champs. Maintenant, il faut dépasser mai. Les premières pluies commencent vers mi-mai ou plutôt fin mai. Avant, le pic c'était en août. Mais maintenant, nous sommes en septembre disons qu'il y a pratiquement un décalage d'un 
mois dans le temps. » (Entretien Individuel\ Directeur de Hydraulique Humaine (DHH) de Korhogo, 25 Février 2010). «Voilà! C'est perturbé maintenant, on ne peut pas dire avec précision que c'est de tant à tant, mais selon les années, il y a des variations » ( Focus group»/Jeunes de Kounontovogo, 06 mai 2010). C’est aussi l'avis de techniciens comme le Directeur Régional (DR) de la SODECI à Korhogo: "Mais il faut noter qu'aujourd'hui tout a changé, on ne maitrise plus les saisons des pluies. Il pleut au moment où l'on ne s'y attend pas et la pluie s'arrête au moment où l'on ne s'y attend pas. Il faut noter qu'il y a un changement de climat. Avant, en avril-mai, il y avait une petite saison des pluies mais aujourd'hui, il peut pleuvoir comme ne pas pleuvoir» (Entretien Individuel\DR SODECI Korhogo, 03 mai 2010).

De façon générale, les enquêtés évoquent également l'avènement d'une nouvelle saison sèche qui n'existait pas avant les années 80 . Aboudramane précise que cette nouvelle saison sèche a été plus forte à partir des années 80 et 83 et qu'elle perturbe beaucoup la production des ignames en particulier («Focus group »/Jeunes de Kounontonvogo/06 mai 2010). En tout état de cause, ces données montrent que les acteurs sont conscients des changements ou perturbations au niveau de la pluviométrie dans leur zone.

Mais au-delà des résultats de la variabilité climatique observée, les populations révèlent d'autres évènements indicateurs du double phénomène de la variabilité et des changements tant climatiques qu'environnementaux à Korhogo. Le Tableau 2 donne un aperçu de ces évènements.

\section{Perceptions des évènements climatiques extrêmes et du paysage en mutation}

Tableau 2: Évènements climatiques extrêmes depuis 1980 et autres changements observé dans l'environnement

\begin{tabular}{|c|c|c|c|}
\hline \multicolumn{3}{|c|}{ Evénements climatiques extrêmes et autres } & \multirow{2}{*}{$\begin{array}{l}\text { Paysage en mutation } \\
\text { Végétation }\end{array}$} \\
\hline $\begin{array}{l}\text { Années de } \\
\text { sécheresse } \\
\text { depuis } 1980\end{array}$ & $\begin{array}{l}\text { Années } \\
\text { d'inondation } \\
\text { depuis } 1980\end{array}$ & $\begin{array}{l}\text { Changement au niveau } \\
\text { de l'harmattan }\end{array}$ & \\
\hline $1983-1984$ & $1980-1981$ & \multirow{3}{*}{$\begin{array}{l}\text { Rigueur du froid en } \\
\text { baisse } \\
\text { Vent plus chaud et plus } \\
\text { sec. } \\
\text { Forte poussière qui } \\
\text { l'accompagne } \\
\text { maintenant alors que } \\
\text { l'on n'en voyait pas } \\
\text { avant. }\end{array}$} & \multirow{3}{*}{$\begin{array}{l}\text { Dégradation du couvert } \\
\text { végétal, des sols et } \\
\text { progression du désert. } \\
\text { Disparition des cours d'eau } \\
\text { et assèchement des } \\
\text { barrages et rivières } \\
\text { Disparition d'espèces } \\
\text { floristiques et fauniques }\end{array}$} \\
\hline 2004-2005 & $\begin{array}{l}\text { Très fortes pluies } \\
\text { en } 2003\end{array}$ & & \\
\hline & $2006-2007$ & & \\
\hline
\end{tabular}


L’analyse des résultats indique deux grandes périodes de sécheresse identifiées en trente ans: en 1983-1984 et ensuite en 2004-2005. Selon les propos des enquêtés, il y a eu en 1984 une grande sécheresse avec un phénomène étrange (nuée de poussières venue du Sahel ou du désert et qui a affecté toute la ville) avec préjudices énormes sur la population: " pénurie d'eau, difficultés à produire, déficit alimentaire et achat de nourritures (en l'occurrence le riz), difficile d'abreuver les animaux qui ont tous dépéri, énormes pertes de riz de bonne qualité et non conservation d'une certaine variété à cause de la sécheresse» ( Focus Group»\Jeunes de Gbondokaha, 11 janvier 2010). Le même constat a été fait également en 2004 avec pratiquement les mêmes effets : pénurie d'eau, tarissement des barrages, des bas-fonds, des rivières et des puits. A cet effet, l'ONG Animation Rurale de Korhogo (ARK) a été cooptée en tant que structure pour ravitailler en eau tous les quartiers de la ville de Korhogo "Cette année là, on allait chercher l'eau jusqu'à Ferkéssédougou du fait qu'au niveau du barrage de Korhogo, il n'y avait pratiquement rien, tout avait tari» (Entretiens Individuels $\backslash$ ONG ARK, 4 mai 2010).

La récurrence du phénomène dans la zone inquiète la population, eu égard à ses conséquences sur elle en général et sur les ressources en eau en particulier. Pour le Directeur Régional (DR) de l'Agriculture à Korhogo, l'on assiste à une installation progressive et définitive du phénomène. Celui-ci soutient que la sécheresse est devenue pratiquement un phénomène courant dans la zone des savanes, dans la mesure où, chaque année au moins, la zone connait quelques mois de sécheresse et de déficit hydrique. Selon lui, l'on observe de plus en plus, non seulement un déficit d'eau chez les plantes mais également, une pénurie d'eau au niveau des ménages. Il note aussi que même en cette année 2010, il n'a pratiquement pas plu tout le mois de juillet et cette situation n'est pas exceptionnelle, elle est de plus en plus récurrente (Entretien Individuel\ Directeur Régional (DR) de l’Agriculture, Korhogo, 11 août 2010).

Si tous les acteurs interrogés ont noté l'installation presque définitive de la sécheresse dans leur zone, ceux-ci ne comprennent pas les pluies diluviennes et abondantes qui inondent des parcelles et causent d'énormes pertes (en matériels et vies humaines).

A ce sujet, un enquêté donne des dates précises des superpositions climatiques en relevant le caractère aberrant de la situation. Il note que, juste avant les différentes périodes de sécheresse, il y a eu une grande pluie qui a inondé les plantations et emporté même des personnes. C'est le cas selon lui « en 1981 déjà, 1980-1981, où il y a eu une grosse inondation et cela a même emporté certaines personnes et en 1984, il y a eu la sécheresse». Parallèlement, en 2000 et en 2003, il y a eu une bonne saison de pluies ou du moins une forte pluie qui a inondé et emporté des parcelles 
de riz déjà repiqué. C’était une grosse perte pour la population qui se devait de tout reprendre. «Mais bizarrement en 2004, il y a eu une sécheresse. Et en 2006-2007, il y a eu encore une inondation qui a eu beaucoup de conséquences, non seulement sur les cultures mais sur l'habitat aussi. Donc il y a beaucoup de personnes qui sont restées sans abri... (Entretiens Individuels\ONG ARK, 4 Mai 2010).

Les conséquences dramatiques de ce phénomène ont été évoquées en termes de dégâts matériels et de pertes en vies humaines: "Nous avons connu des inondations pendant lesquelles l'eau a pénétré dans les maisons après la guerre en 2006-2007. Cela a fait beaucoup de dégâts de l'autre côté du barrage (Haoussabougou), où il y a eu beaucoup de morts d'enfants. Sur la route du quartier Petit-Paris, le pont était envahi d'eau et coupé» (« Focus Group »\Jeunes de Mongaha, mai 2010).

Ce phénomène, plutôt anormal pour certains, commence à devenir normal pour d'autres, si l'on considère les propos qui suivent : « Bon, il faut dire qu'on est habitué à ces deux phénomènes, on commence à s'habituer aux deux phénomènes d'inondations et puis de sécheresse ici dans la zone de Korhogo. » (Entretien Individuel $\backslash$ Directeur Régional (DR) de l'Agriculture, Korhogo, 11 août 2010). Aussi, deux photos du barrage de Korhogo tirées en 2005 et 2007 montrent le paradoxe climatique dans la zone.

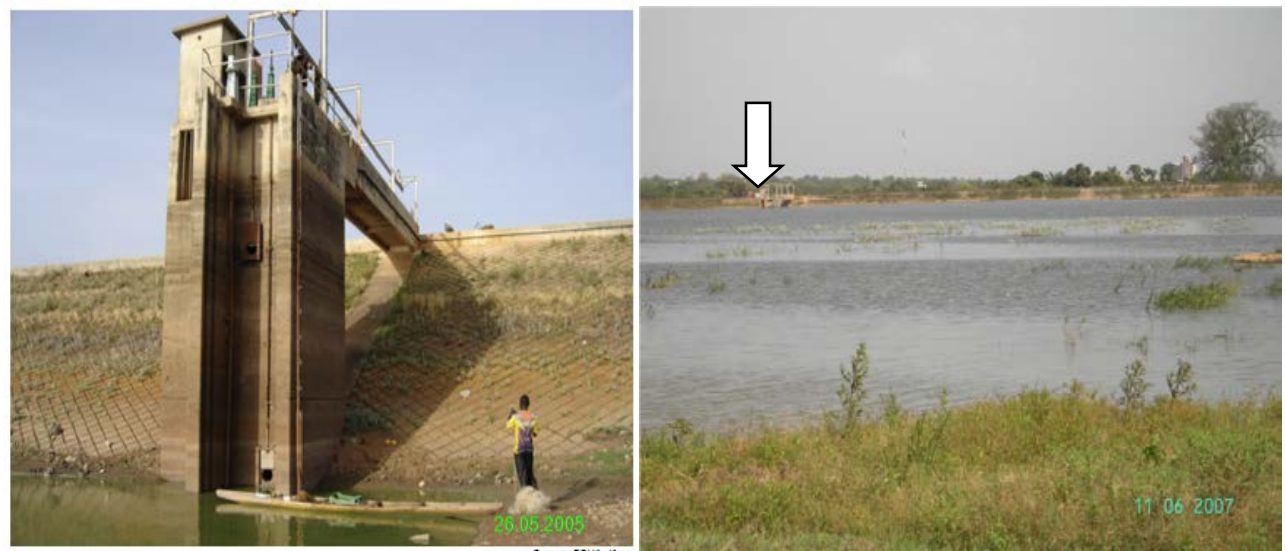

Photos 1 \& 2 : Assèchement et remplissage du barrage lors de la sécheresse de 2004-2005 (26 mai 2005) et de l’inondation de 2006-2007 (11 juin 2007)

La Photo 1 (26 mai 2005), montre qu'il y a eu un assèchement du barrage lors de la sécheresse de 2004-2005. Selon les témoignages des acteurs exerçant autour du barrage, les activités de maraichage se pratiquaient jusque dans le lit du barrage et les enfants se permettaient d'y entrer soit pour pêcher, soit pour jouer. Mais la Photo 2 (11 juin 2007), montre au contraire un barrage complètement rempli, débordant; une situation inattendue et complexe pour la population. 
Par ailleurs, du point de vue des enquêtés, le phénomène a entraîné d'autres perturbations, notamment au niveau de l'harmattan.

Selon eux, l'harmattan a subi un changement indéniable, s'expliquant, pour les uns par le manque de pluies et pour d'autres par la disparition de la végétation du fait de l'exploitation abusive de la flore. « Avant, il faisait plus froid à cause de la pluie, mais actuellement, il y a plus de poussières. En approchant le lit d'un bas-fond, il faisait de plus en plus froid, mais actuellement on peut même aller y construire une maison. Pour moi, l'harmattan est lié à la pluie. Quand il pleut beaucoup, il fait aussi froid. De nos jours, ce qui est développé, c'est plus le vent et la poussière, mais il y a moins de fraîcheur» ("Focus Group »\Jeunes de Mongaha, mai 2010).

Concernant la durée et la période de l'harmattan avant et après les années 80, les enquêtés se disent embarrassés car tout a changé. Selon eux, avant, décembre était le mois où le froid commençait pour aller jusqu'en janvier-février. Mais le vent pouvait commencer à partir de fin janvier et traîner jusqu'en mars, début avril et cela ne durait pas. Maintenant, il y a une confusion totale, un bouleversement qu'on ne peut expliquer.

Ce bouleversement, selon le Directeur Régional de l’ANADER de Korhogo, agit sur la production de certaines cultures vivrières. Dans ses propos, il revient non seulement sur les changements au niveau de l'harmattan mais particulièrement, sur ses conséquences au niveau de l'agriculture. Il soutient que l'harmattan qui s'installe maintenant, n'est pas forcément favorable à l'agriculture au Nord. Selon lui, pendant l'harmattan, il ne pleut certes pas mais il y a cette précipitation atmosphérique qui est la rosée du matin. Cette rosée est une autre forme de pluviosité qui profite à certaines plantes. Mais, comme l'harmattan n'est plus intense, cette forme de pluviosité n'est plus ou très peu observée. Et pourtant, il y a certaines cultures qui, en fonction de l'intensité du froid, produisent. Mais, puisqu'aujourd'hui le froid n'est plus très perceptible, cela a un effet sur ces spéculations. C'est le cas, par exemple, de l'oignon qui bulbe surtout quand il fait très froid et aussi, d'autres cultures comme le manguier, qui donne très bien quand il fait très froid (Entretiens Individuels $\backslash$ DR de l'ANADER, 4 mai 2010).

Les changements dans l'environnement à Korhogo touchent également la végétation. Selon le constat des acteurs, la zone de Korhogo connaît actuellement une dégradation avancée de sa végétation, des sols et une progression du désert. Une situation caractérisée par la disparition des cours d'eau et l'assèchement des barrages, rivières, bas-fonds et puits. Les exemples sont légion: bas-fonds de Torgo à Nissonkaha, exploitables à 95 ha avant les années 1980, l'est maintenant à seulement 55 ha en saison des pluies et les 95 ha, inexploitables en saison sèche. Au niveau des puits, ceux 
de Dokaha situé à $6 \mathrm{~km}$ de Korhogo, montre que sur 32 puits recensés en 2010, seulement 16 sont encore exploitables en saison des pluies, 08 sont utilisables en saison sèche et 08 complètement abandonnés. Egalement, au quartier Sonzoribougou à Korhogo, sur 45 puits recensés, seulement 23 sont utilisables en saison sèche. Concernant les barrages et rivières, le barrage de Zangaha (village à $15 \mathrm{~km}$ de Korhogo) très en crue autrefois, est carrément sec aujourd'hui. "Banaloho », rivière de Lognonkaha (15 km de Korhogo), permanente autrefois, est traversée aujourd'hui à pieds secs. Les photos 3 et 4 attestent de la situation vécue et décrite par les enquêtés.

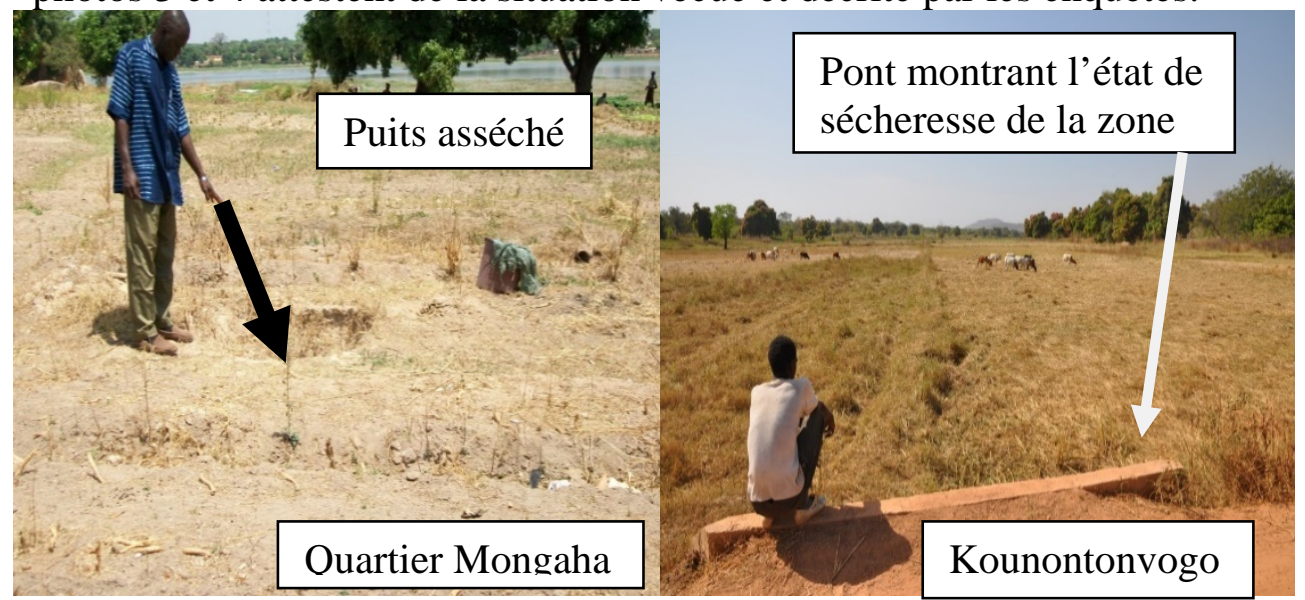

Photos 3 \& 4: Assèchement des cours d'eau (Source : Boko, 2013)

Par ailleurs, nombreuses sont les espèces fauniques et floristiques qui ont disparu ou qui sont en voie de disparition. Ce sont, par exemple, des animaux comme l'éléphant, les hyènes, les singes et certains oiseaux comme les charognards, les vautours (en Sénoufo « katchohoho ») qui sont actuellement introuvables dans la zone. Il y a également un type de poisson que l'on ne retrouve plus, du fait de l'assèchement des sources d'eau. Ce type de poisson appelé « djégourou » ou bien encore " gourou » (en langue Sénoufo) produisait une décharge électrique quand l'on le touchait. Au niveau floristique, plusieurs espèces sont concernées. 
Tableau 3: Espèces disparues ou en disparition

\begin{tabular}{|c|c|c|}
\hline $\begin{array}{l}\text { Noms Sénoufo des } \\
\text { plantes }\end{array}$ & Noms scientifiques & Usages des plantes \\
\hline « Lokpinninmin » & $\begin{array}{l}\text { Mimusops Andongensis } \\
\text { (Sapotaceae) }\end{array}$ & $\begin{array}{l}\text { Vertus thérapeutiques: soigne les maladies de } \\
\text { la peau } \\
\text { Vertus culturelles: sève utilisée pour souder le } \\
\text { gros tambour qui accompagne les « balafons » } \\
\text { (xylophone africain, instrument des musiciens } \\
\text { professionnels malinkés) }\end{array}$ \\
\hline « Sedjodigue » & $\begin{array}{l}\text { Diospyros Mespiliformis } \\
\text { (Ebenaceae) }\end{array}$ & $\begin{array}{l}\text { Vertus alimentaires: fruits consommés, } \\
\text { Vertus thérapeutiques: soigne diarrhées et } \\
\text { vomissements }\end{array}$ \\
\hline « Kasseremi » & $\begin{array}{c}\text { Uapaca Togoensis } \\
\text { (Euphorbiaceae) }\end{array}$ & $\begin{array}{c}\text { Vertus thérapeutiques: utilisée contre } \\
\text { vomissements et toux }\end{array}$ \\
\hline « Djegan » & $\begin{array}{c}\text { Zanthoxylum } \\
\text { Zanthoxyloides } \\
\text { (Rutaceae) }\end{array}$ & $\begin{array}{l}\text { Vertus thérapeutiques: racines soignent maux } \\
\text { de tête et cure-dent utilisé contre les maux de } \\
\text { dents }\end{array}$ \\
\hline « Kadjami » & $\begin{array}{l}\text { Combretum Molle } \\
\text { (Combretaceae) }\end{array}$ & $\begin{array}{l}\text { Vertus thérapeutiques: décocté aqueux des } \\
\text { feuilles fraîches ou sèches calme les } \\
\text { céphalalgies par voie orale; soigne également } \\
\text { diarrhées, maux de ventre, paludisme et } \\
\text { problèmes respiratoires }\end{array}$ \\
\hline $\begin{array}{c}\text { «Djirime » ou } \\
\text { «Djirimin » }\end{array}$ & $\begin{array}{l}\text { Leptadenia Hastata } \\
\text { (Asclepiadaceae) }\end{array}$ & Vertus culinaire: sert à faire la sauce \\
\hline
\end{tabular}

(Source: Boko, 2013)

Pour les populations, la disparition progressive de ces espèces constitue un réel problème car, comme l'indique le tableau ci-dessus, certains de ces arbres avaient des vertus thérapeutiques et constituent une réelle perte pour la médecine traditionnelle. Malheureusement, aussi bien les guérisseurs que les populations n'ont aucun moyen d'en trouver ou alors il faut parcourir des dizaines, voire des centaines de kilomètres pour en avoir. Même les artistes sculpteurs et tous ceux qui travaillent avec des plantes ou arbres spéciaux ont des difficultés dans l'exercice de leurs métiers.

Les populations déplorent également la dégradation progressive des forêts et bois sacrés. Cette dégradation des bois sacrés, particulièrement, constitue une source d'angoisse pour les populations enquêtées du fait de son importance et de ces différents rôles dans la société Sénoufo (rôle culturel de préservation de l'identité du peuple Sénoufo, socialisation et éducation, rôle médical en termes de soin et conservation des plantes médicinales, rôle spirituel et mystique de protection de la population). Selon eux, certains bois sacrés, auparavant très touffus et autour desquels on n'osait pas passer, comme celui de Gbandokaha, un des villages de la zone, sont aujourd'hui dans un état de dégradation avancée. Pour eux, le manque de pluies ne permet pas un renouvellement systématique des gros arbres qui soutenaient ces forêts. 


\section{Discussion: défis face à ce phénomène}

A l'analyse, les données historiques sur la pluviométrie dans la zone de Korhogo indiquent deux grandes saisons (SEDES, 1967) reparties dans l'année de façon égale: six mois de saison sèche (Octobre à Mars) et six mois de saison pluvieuse (Avril à Septembre). Les résultats des entretiens approfondis, coïncident avec ceux de Synali (1978), concernant la grande irrégularité intra-annuelle de la pluviométrie. Ainsi, l'on note une perturbation dans la durée et la période des saisons qui révèle un raccourcissement de la saison pluvieuse au profit de la saison sèche qui s’allonge (Kouakou et al., 2013, M’Bra, 2013, Anon, 2012), en phase avec la baisse des précipitations qui s'est intensifiée en Côte d'Ivoire comme dans les autres pays du golfe de Guinée au cours des années 1980 et 1990 (Noufé, 2011, Brou et al., 2005; Paturel et al., 1995; Le Borgne,1990).

Cette baisse de la pluviométrie et l'enregistrement en Afrique de l'Ouest d'une des sécheresses les plus importantes au cours des années 19701990 (d’Orgeval, 2008), sont les signes de la variabilité du climat dont la fréquence et l'intensité ont augmenté durant les 30 dernières années (Deynès, 2008); occasionnant en particulier un fort déficit hydrique dans la zone de Korhogo (Koné et al., 2007 et Brou et al., 1999). La dégradation du couvert végétal, la disparition des cours d'eau et l'assèchement des barrages et rivières dans la zone d'étude confirme ce fort déficit hydrique. Ce résultat est en phase avec celui de Brou et al. (2005) sur les conséquences de la baisse de la pluviométrie dans la zone. Leur étude montre que les effets de la récession pluviométrique récente sur les ressources en eau se traduisent, pendant les longues périodes de sécheresse, par une baisse de plus en plus fréquente, du débit des cours d'eau et du niveau des nappes phréatiques, allant jusqu'à l'assèchement, le tarissement des rivières et marigots, compromettant ainsi les activités agricoles des acteurs. Ce fut le cas du barrage d'approvisionnement en eau potable (AEP) de la ville de Korhogo qui, malgré sa capacité de $10000000 \mathrm{~m} 3$, a connu un assèchement total pendant la sécheresse de 2005 (N’krumah et al., 2014).

Une situation qui n'est pas faite pour aider la population si nous considérons les rapports privilégiés de ce peuple avec son environnement naturel. Rappelons que le peuple Sénoufo est particulièrement attaché à la terre et à la nature de par ses pratiques cultuelles et culturales. La pluviométrie est en effet, le facteur prépondérant qui conditionne et rythme les activités agricoles et partant, la vie même des paysans Sénoufo (Sinaly, 1978). Ainsi, de la variabilité climatique à la problématique des ressources en eau en passant par la disparition d'espèces animales et végétales, l'étude révèle trois grands problèmes que connaît ou pourrait connaître la zone de Korhogo du fait de sa vulnérabilité climatique. 


\section{Le premier problème est relatif à la dégradation des ressources}

Il a été montré que la zone de Korhogo subit une sécheresse continue accompagnée de la dégradation de ses sols, la désertification, l’assèchement de ses cours d'eau et la disparition de ses espèces floristiques et fauniques. Ce résultat coincide avec celui de Deynès (2008), qui montre que la probabilité est forte que le changement climatique soit accompagné par la dégradation des ressources, avec une désertification et des sécheresses accrues qui peuvent conduire à une baisse de la production agricole. Cette dégradation des ressources est un véritable frein au développement des activités agricoles et par la même occasion, à l'épanouissement des populations qui ont pour activité principale l'agriculture. En plus de la dégradation de ses ressources, l'étude met également en exergue la pression foncière et démographique dans la zone, évoquée dans l'étude de Zabgaï (2006). A Korhogo, se posent également d'autres problèmes identifiés par l'étude. Il s’agit des conflits liés à la pénurie d'eau et du problème de la sécurité alimentaire.

\section{Le second problème est celui des conflits liés à la pénurie d'eau}

La question de la disponibilité de l'eau se pose à Korhogo, avec acuité surtout pendant la saison sèche. Les populations se plaignent de plus en plus du manque d'eau pour la consommation, pour les activités agricoles, pour l'élevage, etc.

Cette pénurie d'eau est à la base de nombreux conflits entre les communautés et constitue un motif de migration de la zone de Korhogo vers d’autres zones plus humide. Ces conflits générés par la disponibilité des ressources en eau sont évoqués dans l'étude de Niasse et al. (2004), qui relève qu’il existe une relation forte entre les sécheresses et la probabilité de conflits de haute intensité.

\section{Le troisième problème est lié à la sécurité alimentaire}

Les cultures vivrières subissent une baisse de rendement, les périodes de soudures deviennent de plus en plus longues, les populations se plaignent de l'insuffisance et du manque de nourritures ; des éléments qui augurent de l'insécurité alimentaire à Korhogo. Une étude sur l'évaluation approfondie de la sécurité alimentaire des ménages ruraux en Côte d’Ivoire réalisée par le Programme Alimentaire Mondial (PAM, 2009), Bureau de pays, Côte d'Ivoire, montre que l'insécurité alimentaire est persistante dans la région des Savanes à 17,3\%. D’autre études montrent combien la dégradation des conditions propices à l'agriculture pourrait conduire à la détérioration de la sécurité alimentaire, à une recrudescence des famines, à la dépendance accrue aux importations alimentaires et à l'instabilité des prix (Deynès, 2008) du fait de la péjoration du climat (Niasse, 2007). 


\section{Conclusion}

L'étude sur la variabilité climatique à Korhogo, permet de faire quatre constats :

a) Une perturbation dans les périodes et durées des saisons durant les trente dernières années et un raccourcissement de la saison des pluies au profit de la saison sèche

b) Korhogo a connu des périodes de grandes sécheresses en 19831984 et ensuite, en 2004-2005. Puis, des périodes de pluies abondantes en 1980-1981, en 2003 et en 2006-2007.

c) Une dégradation des ressources (dégradation du couvert végétal, aridité des sols, avancée progressive du désert, disparition des espèces floristiques et fauniques, assèchement des sources d'eau (rivières, marigots, bas-fonds, puits) surtout en saison sèche.

d) De nombreux problèmes sociaux spécifiques tels que les conflits liés à la pénurie d'eau, l’insécurité alimentaire, les pressions démographiques et foncières, les migrations et exodes rurales, les pratiques difficiles de la médicine traditionnelle. Ces problèmes sociaux engendrent d'autres problèmes collatéraux tels que l'augmentation du coût de la vie, la déscolarisation, la délinquance, le chômage, les problèmes de santé (anxiété par rapport à l'avenir) du fait de la baisse des rendements liée aux perturbations climatiques. Une situation d'inquiétude et de stress générale qui appelle à une plus grande conscience écologique chez la population.

En somme, la population vit dans une situation de précarité sociale, hydrique et environnementale qui appelle à davantage d'intérêts de la part des autorités politiques. Ainsi,

Le préalable dans cette zone, c'est communiquer suffisamment sur le changement climatique, ses causes et ses conséquences pour une meilleure connaissance du phénomène ensuite,

Faire une éducation environnementale pour inculquer à la population des valeurs de conservation et de protection de l'environnement en vue d'un renforcement de la conscience écologique,

Mener des actions concrètes de sensibilisation pour le reboisement de la zone,

Encourager les populations à diversifier ses activités en privilégiant les activités génératrices de revenus pour supporter les périodes de soudures qui s’allongent au fil des années,

Créer des comités villageois chargés de surveiller scrupuleusement et de noter les périodes et durées des saisons, de contrôler le comportement ou la résistance des cultures au fil des années afin de privilégier et d'adopter les cultures désormais favorables à la zone,

Réhabiliter les barrages hydro-agricoles pour conserver et mieux maîtriser la circulation de l'eau dans la zone. 
Ces suggestions sont des pistes qui devraient permettre une amélioration des conditions du milieu et de la vie de la population face au phénomène de la variabilité climatique.

\section{Remerciements}

Les auteurs adressent leurs remerciements au Centre de Recherches pour le Développement International (CRDI) et au Centre Suisse de Recherches Scientifiques en Côte d'Ivoire (CSRS) à travers le Programme dit CSRS-CRDI-CC. Remerciements également aux Programmes RIPIECSA et le "National Center of Competence in Research North -South (NCCR North-South) ainsi qu'à tous les membres de l'équipe projet CSRS-CRDICC de Korhogo pour leurs contributions.

\section{References :}

Anon S. R. F.: Contribution à l'analyse des impacts des Changements Climatiques sur la production Agricole de la zone périurbaine de Korhogo. Diplôme d'Agronomie Approfondie (DAA), Ecole Supérieure d'Agronomie (ESA) de l'Institut National Polytechnique Félix-Houphouët Boigny (INPHB) de Yamoussoukro: Côte d’Ivoire, 2012

Brou Y.T., Akindès F., Bigot S. : La variabilité climatique en Côte d’Ivoire : entre perceptions sociales et réponses agricoles. Cahiers Agricultures, 2005 Kouakou Yao Etienne, Koné Brama, Cissé Guéladio, Djè Kouakou Bernard, N’Go Yao Alexis, Konaré Abdourahamane, Savané Issiaka. Environmental Vulnerability Index: application to the White Bandama Bassin in the northern Côte d'Ivoire. International Journal of Conservation Sciences, 2013. M'Bra K. R.: Approvisionnement en eau potable, déficit d'assainissement et risques sanitaires associés dans un contexte de variabilité climatique à Korhogo (Nord Côte d'Ivoire). Diplôme d'Etudes Approfondies (DEA). Université Félix Houphouët Boigny : Côte d’Ivoire, 2013

Ministère de l'environnement et de la forêt. Diversité biologique de la Côte d'Ivoire, 1999

Le diagnostic du Livre Blanc de l'Environnement de Côte d’Ivoire, 1995.

Ministère de l'Environnement, de la Construction et de l'Urbanisme (MECU). Environnement et développement, rapport National sur l'état de l'environnement en Côte d'Ivoire, 1991.

Koné, I. : Peut-on encore éviter la dérive écologique en Côte d’Ivoire? Communication orale, conférence scientifique. CSRS, Abidjan, 2011.

N’Guessan E., Dibi N. H., Bellan M., Blasco F.: Pression anthropique sur une réserve forestière en Côte d’Ivoire. Télédétection, 2006.

N’krumah TASR, Koné B, Tiembré I, Mbaye I, Tanner M, Cisse G.: Variabilité climatique et incidence de la méningite cérébro-spinale dans le district sanitaire de Korhogo (Nord de la Côte d’Ivoire). Environ Risque 
Santé, 2014

Yao A. C.Y.: Pratiques paysannes et dynamiques de la biodiversité dans la forêt classée de Monogaga (Côte d’Ivoire). Thèse de doctorat, Département Hommes Natures Sociétés, Muséum National d’Histoire Naturelle. Paris, 2005.

Commission Européenne: Environnement et changement climatique en Côte d'Ivoire. Côte d’Ivoire - Communauté européenne. Document de stratégie pays et Programme indicatif national pour la période 2008-2013, 2006.

Sedes: Région de Korhogo: Etude de développement socio- économique. Rue de LILLE- Paris-7e, 1967.

Coulibaly S.: Le paysan sénoufo. Les Nouvelles Éditions Africaines, Abidjan-Dakar, 1978

Paturel J.E., Servat E., Kouamé B., Masson J., Lubes H.: La Sécheresse en Afrique de l'Ouest non sahélienne (Côte d’Ivoire, Togo, Bénin). Sécheresse, 1995

Le Borgne J.: La dégradation actuelle du climat en Afrique entre Sahara et Équateur. In: Richard JF, ed. La dégradation des paysages en Afrique de l'Ouest. Paris ; Dakar : Aupelf; Coopération Française; Union internationale pour la conservation de la nature (UICN); Orstom-ENDA- TM, Documentation française, 1990

Deynès, F.: Les impacts du changement climatique en Afrique conflictualité associé. Mémoire dans le cadre du séminaire « Prospectives sur l'Afrique », 2008

Koné M., Aman A., Adou Y. C. Y., Coulibaly L., N’guessan K. E.: Suivi diachronique par télédétection spatiale de la couverture ligneuse en milieu de savane soudanienne en Côte d’Ivoire. Revue Télédétection, 2007

Programme Alimentaire Mondial (PAM) Côte d'Ivoire: Evaluation approfondie de la sécurité alimentaire des ménages ruraux en Côte d'Ivoire, rapport final, 2009

Niasse M,: Eléments de stratégie régionale d'adaptation au changement climatique basée sur l'approche de partage des risques - Afrique de l'ouest. Programme Adaptation au Changement Climatique en Afrique, 2007.

Noufé Dabissi. Changements hydro-climatiques et transformations de l'agriculture : l'exemple des paysanneries de l'Est de la Côte d'Ivoire. THĖSE Pour l'obtention du grade de Docteur de l'Université de Paris 1. Spécialité : Géographie, 2011.

Hubert Sery Zagbaï, Fabio Berti, Philippe Lebailly. Impact de la dynamique cotonnière sur le développement rural. Étude de cas de la région de Korhogo, au Nord et au Centre de la Côte d'Ivoire. Biotechnol. Agron. Soc. Environ., 2006 